Article

\title{
GmIDL2 $a$ and GmIDL4a, Encoding the Inflorescence Deficient in Abscission-Like Protein, Are Involved in Soybean Cell Wall Degradation during Lateral Root Emergence
}

\author{
Chen Liu, Chunyu Zhang, Mingxia Fan, Wenjuan Ma, Meiming Chen, Fengchun Cai, \\ Kuichen Liu and Feng Lin * \\ College of Bioscience and Biotechnology, Shenyang Agricultural University, Shenyang 110086, China; \\ liuchen@syau.edu.cn (C.L.); cy_zhangsyau@outlook.com (C.Z.); syndlc@outlook.com (M.F.); \\ 20152166@stu.syau.edu.cn (W.M.); 20162164@stu.syau.edu.cn (M.C.); 2017220160@stu.syau.edu.cn (F.C.); \\ 2017220164@stu.syau.edu.cn (K.L.) \\ * Correspondence: 2001500072@syau.edu.cn; Tel.: +86-24-8848-7163
}

Received: 7 June 2018; Accepted: 22 July 2018; Published: 2 August 2018

\begin{abstract}
The number of lateral roots (LRs) of a plant determines the efficiency of water and nutrient uptake. Soybean is a typical taproot crop which is deficient in LRs. The number of LRs is therefore an important agronomic trait in soybean breeding. It is reported that the inflorescence deficient in abscission (IDA) protein plays an important role in the emergence of Arabidopsis LRs. Previously, the genes which encode IDA-like (IDL) proteins have been identified in the soybean genome. However, the functions of these genes in LR development are unknown. Therefore, it is of great value to investigate the function of $I D L$ genes in soybean. In the present study, the functions of two root-specific expressed IDL genes, GmIDL2a and GmIDL4a, are investigated. The expressions of GmIDL2a and GmIDL4a, induced by auxin, are located in the overlaying tissue, where LRs are initiated. Overexpression of GmIDL2a and GmIDL4a increases the LR densities of the primary roots, but not in the elder root. Abnormal cell layer separation has also been observed in GmIDL2a- and GmIDL4a-overexpressing roots. These results suggest that the overlaying tissues of GmIDL2a- and GmIDL4a-overexpressing roots are looser and are suiTable for the emergence of the LR primordium. Further investigation shows that the expression of some of the cell wall remodeling (CWR) genes, such as xyloglucan endotransglucosylase/hydrolases, expansins, and polygalacturonases, are increased when GmIDL2a and GmIDL4a are overexpressed in hairy roots. Here, we conclude that GmIDL2a and GmIDL4a function in LR emergence through regulating soybean CWR gene expression.
\end{abstract}

Keywords: soybean; IDA-like protein; cell wall remodeling genes; lateral root development

\section{Introduction}

Root system architecture represents a key adaptability, enabling plants to cope with abiotic stresses such as drought, nutrient deficiency, and salinity, and is therefore very important for plant growth and development. The degree of root branching, determined by LR formation, influences the anchorage of plants to the soil, as well as nutrient acquisition and the efficiency of water uptake. The capacity of the root system to fulfill these functions is highly dependent on its architecture. Many monocotyledonous plants, such as maize (Zea mays) and rice (Oryza sativa), have adventitious roots, whereas dicotyledonous crops, such as cotton (Gossypium hirsutum) and soybean (Glycine max), have a primary root that repeatedly branches to generate several orders of LRs [1,2]. The degree of LR development is therefore a vital agronomic trait in crop breeding. 
LRs originate from triplets of adjacent xylem pole pericycle cell pairs at regular intervals, named LR founder cells [2,3]. LR founder cells undergo several rounds of anticlinal divisions to form a single layer of primordium, consisting of small cells with equal length [4-6]. The subsequent rounds of divisions occur periclinally and create a two-layered lateral root primordium (LRP). Further anticlinal and periclinal divisions produce a dome-shaped LRP that divides progressively as the primary root meristem. This process is associated with auxin transport [7-10], auxin signaling [11,12], and oscillations of the auxin responses [9].

Organ emergence is another important event which restricts LR development. The LRPs are embedded within the overlaying tissue of the primary root. Therefore, to emerge, LRPs must first penetrate their parent tissues, comprising an endodermis, cortex, and epidermis. Plant cells are constrained by rigid cell walls consisting of cellulose, hemicellulose, and pectin, of which pectin is the major chemical component of the plant cell wall. The emergence of LRs and cell separation are highly coordinated. For one thing, the penetration of the LRP involves the initial cell wall loosening effected by enzymes such as xyloglucan endotransglucosylase/hydrolases (XTHs) and expansins (EXPs) [13,14]. The incompact cell walls facilitate the entrance of polygalacturonases (PGs), an important cell wall degradation enzyme which hydrolyzes pectins in cell separation events $[13,14]$. On the other hand, the overlaying tissue can disrupt the morphogenesis of the LRP [15].

Many biological processes are associated with cell wall loosening and degradation. Floral organ abscission happens after pollination and is controlled by the peptide IDA, which functions through its receptor leucine-rich repeat receptor-like kinases HAESA (HAE) and HAESA-LIKE2 (HSL2) [16,17]. The mutation of the IDA gene is associated with a deficiency in floral organ abscission in Arabidopsis and Lupinus luteus [18-20]. The mutant phenotype can be complemented by IDL proteins from other species [21,22]. Overexpression of IDA induces ectopic abscission and increases the number of rounded abscission zone (AZ) cells [23]. Microarray data shows that the IDA-HAE/HSL2 signaling module is involved in the regulation of CWR gene expression [13]. Similarly, the emergence of LRPs is dependent on cell wall dissolution. LR density is decreased in the ida mutant due to the constraint of overlaying the endodermal, cortical, and epidermal tissues [24]. Moreover, the IDL gene is associated with bacterial infection. Arabidopsis IDA6 is upregulated significantly when the plant is suffering from attacks of the bacterial Pseudomonas syringae pv. tomato (Pst) DC3000 [25]. IDL sequences are also found in the genomic sequences of Meloidogyne spp., which are globally deleterious pathogens of plants. The root-knot nematodes can secrete a functional IDA mimic to facilitate its infection to the host [26].

Previously, 12 GmIDL genes have been identified in the soybean genome, in which GmIDL2a and GmIDL4a were highly expressed in the root [27]. Moreover, the expression levels of GmIDL2a and GmIDL4a in the elder root were higher than those in root tips [27]. However, the functions of these two genes in LR development are still unknown. In the present study, we used a reversed genetics strategy to further investigate the functions of GmIDL2a and GmIDL4a in soybean LR development. We found that the expressions of GmIDL2a and GmIDL4a were induced by auxin. The overexpression of GmIDL2a and GmIDL4a can accelerate the emergence of LRPs in primary roots. The LR densities in 35S::GmIDL2a and 35S::GmIDL4a elder roots were not affected. Meanwhile, abnormal cell layer separation was observed in elder roots when GmIDL2a and GmIDL4a were overexpressed. Furthermore, the expressions of some of the CWR genes were increased when GmIDL2a and GmIDL4a were overexpressed. Our results enhanced the understanding of soybean LR emergence and indicated a potential use of these genes in soybean root architecture improvement.

\section{Results}

\subsection{GmIDLs Are Highly Similar and Redundant in the Soybean Genome}

The EPIP, the functional motif of IDA/IDL, is sufficient for IDA to play a role in floral organ abscission in Arabidopsis [28]. In previous reports, the soybean IDL genes were identified through 
BLAST searching with the use of an IDA protein sequence (NP564941) [27]. The results showed that a total of 12 proteins containing a similar motif to EPIP have been identified in the soybean genome [27]. The protein sequences are shown in Figure 1A. The twelve IDL proteins, named GmIDL1a to GmIDL6b, include putative signal peptides in their $\mathrm{N}$-terminal variable regions immediately after the signal peptides and a C-end in their sequences (Figure 1A). The protein sequences of GmIDLs were very similar, indicating the redundant function of these genes.

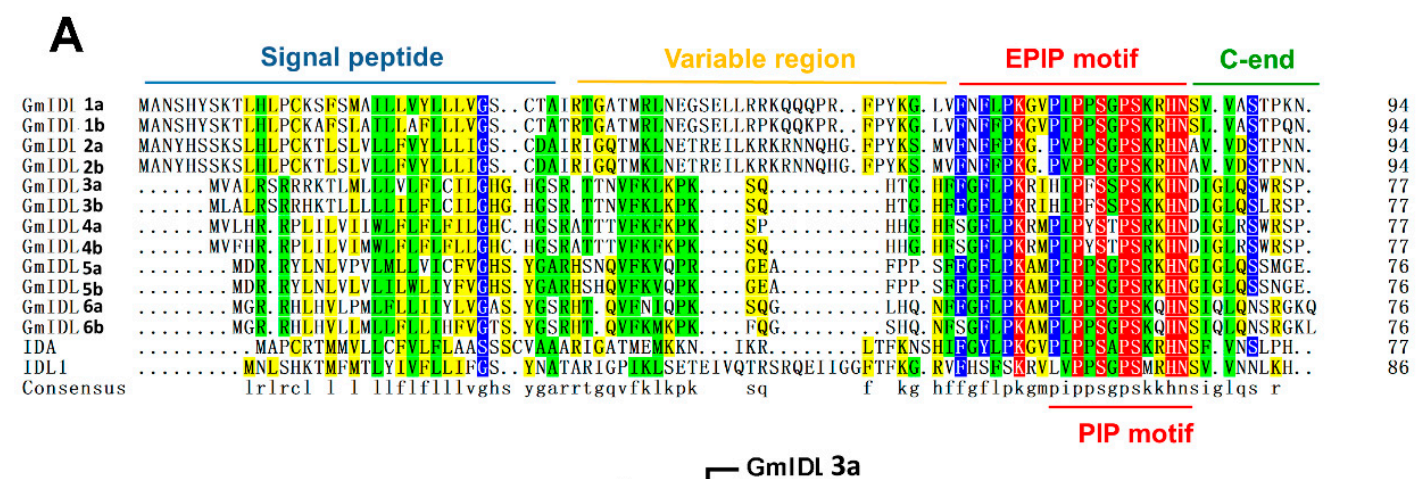

B

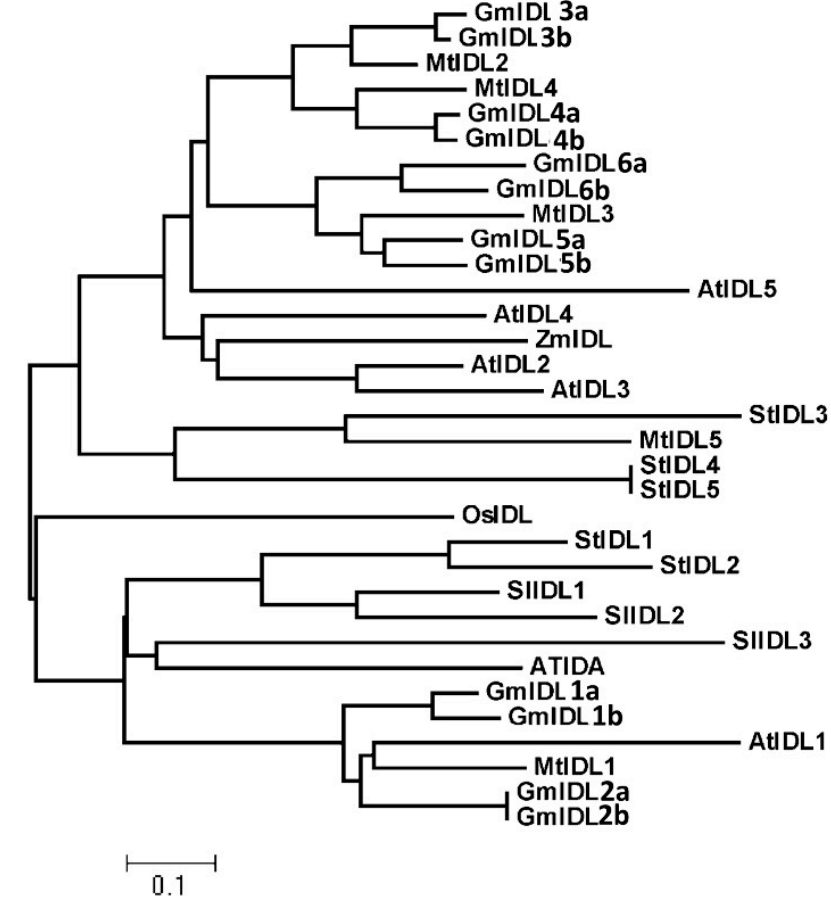

Figure 1. Protein sequence alignment and phylogenetic tree analysis of GmIDLs. (A) Alignment of soybean GmIDL proteins; and (B) phylogenetic tree analysis of IDL proteins in rice (OsIDL), maize (ZmIDL), tomato (SIIDL), potato (StIDL), lucerne (MtIDL), and soybean (GmIDL). IDL, IDA-like protein; EPIP, the functional motif of IDA/IDL protein.

To clarify the evolutionary relationship, we further searched for the IDL proteins in rice, maize, potato, tomato, and lucerne using the same approach. The evolutionary relationship of the twelve soybean, one rice, one maize, five lucerne, three tomato, five potato, and six Arabidopsis IDL proteins were displayed in the dendrogram (Figure 1B). The 12 GmIDLs were divided into two groups (Figure 1B). Group one included GmIDL1a to GmIDL2b, which shared the highest similarities with MtIDL1, IDL1, IDL, and SIIDL3, and had relatively high similarities with StIDL1, StIDL2, SIIDL1, SIIDL2, and OsIDL. Group two included GmIDL3a to GmIDL6b. In the other eight GmIDLs, GmIDL3a and GmIDL3b had the highest similarities with MtIDL2, and GmIDL4a and GmIDL4b were most similar to MtIDL4 (Figure 1B). GmIDL5a to GmIDL6b had the highest similarities with MtIDL3, 
AtIDL5, AtIDL4, ZmIDL, AtIDL2, and AtIDL3, respectively (Figure 1B). According to the results, we found that GmIDL genes were highly similar paralogous genes. These results also suggested that functional redundancy might exist in GmIDL genes.

\subsection{The Expression of GmIDL2a and GmIDL4a Is Induced by Auxin and Located in the Cell Layers Embedding LRP}

To identify the functional gene in soybean LR development within these 12 GmIDLs, total RNA was extracted from 3-4 cm soybean roots for RT-PCR assay. The results showed that GmIDL2a, GmIDL2b, and GmIDL4a were expressed in roots, in which GmIDL2a and GmIDL4a were highly expressed (Figure 2A). The RT-PCR results indicated potential functions of these two genes in LR development. These results were consistent with previous reporting [27]. Therefore, GmIDL2a and GmIDL4a were selected for further research.

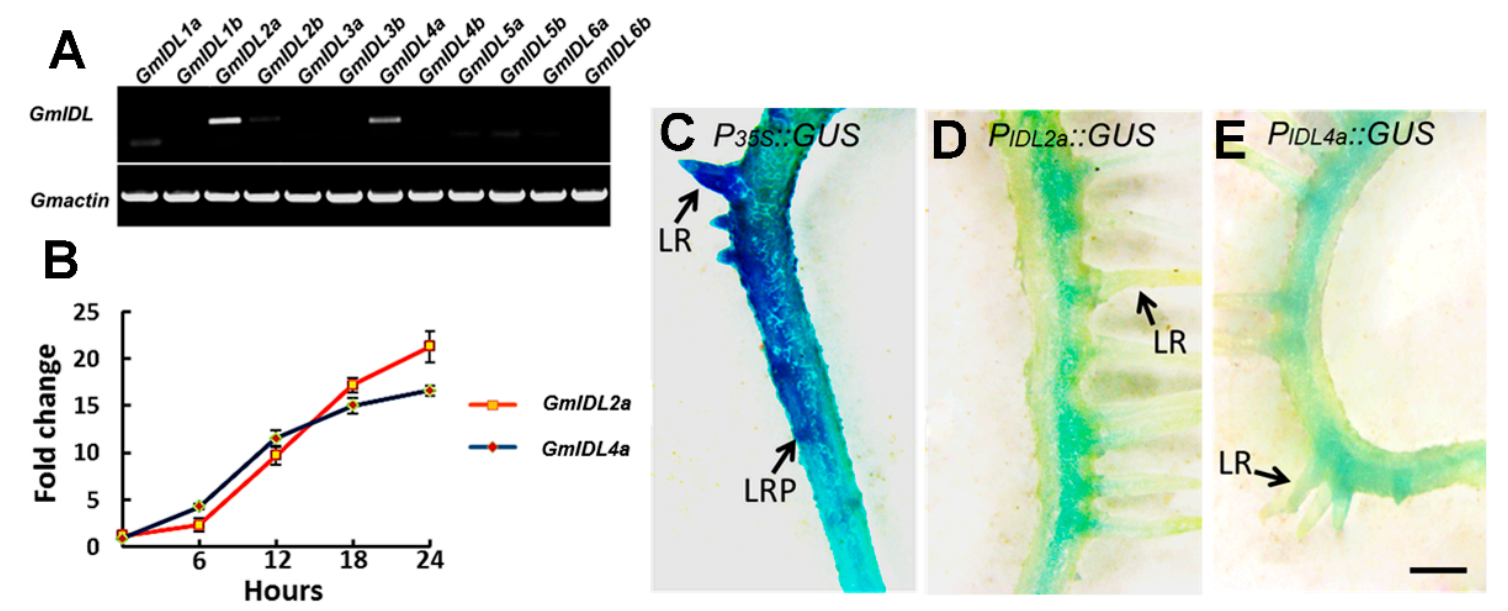

Figure 2. The expression of GmIDL genes in soybean roots. (A) RT-PCR analysis of GmIDLs in soybean roots; (B) relative expression of GmIDL2a and GmIDL4a under auxin treatments; (C-E) GUS histochemical staining assay of $P_{355}:: G U S ;(C) P_{I D L 2 a}: \because G U S$ and $P_{I D L 4 a}:: G U S$ hairy roots under normal conditions. LR, lateral root; LRP, lateral root primordium; scale bar $=2 \mathrm{~mm}$.

Because GmIDL2a and GmIDL4a were proposed to be associated with LR emergence [27] and auxin has been reported to play an important role in LR development [2], we therefore tested the effect of auxin on GmIDL expression. The roots were treated in $1 \mu \mathrm{M}$ IAA in MS medium for 6 to $24 \mathrm{~h}$. The expression of GmIDL2a and GmIDL4a was increased after IAA treatment and acquired sharp increases after $6 \mathrm{~h}$. Before $12 \mathrm{~h}$, the expression of GmIDL4a was higher relative to GmIDL2a, and peaked after $18 \mathrm{~h}$ of treatment (Figure 2B). However, the expression of GmIDL2a was still increased after $18 \mathrm{~h}$ (Figure 2B). These results suggested that the expression of GmIDL2a and GmIDL4a was induced by auxin.

Furthermore, the expression patterns of GmIDL2a and GmIDL4a in roots were investigated using promoter::GUS constructs. Transgenic hairy roots harboring $P_{G m I D L}:: G U S$ constructs were obtained from Agrobacterium rhizogenes-mediated transformation. Hairy roots harbouring the corresponding constructs were used for GUS histochemical staining assay. The GUS activity was detected in emerged LRs and all the cell layers of P35S::GUS primary roots (Figure 2C). In contrast, the promoter activities of GmIDL2a and GmIDL4a in hairy roots were only detected in one side of the cell layers where LRP emerged, and not in the other side (Figure 2D,E). The expression patterns of GmIDL2a and GmIDL4a indicated the potential functions in LR development. 


\subsection{GmIDL2a and GmIDL4a Facilitate LRP Penetration through the Overlaying Cell Layers during LR Emergence}

To investigate the functions of GmIDL2a and GmIDL4a in soybean LR development, the genes GmIDL2 $a$ and GmIDL4a were overexpressed in soybean hairy roots, driven by the CaMV $35 S$ constitutive promoters. 35S::GFP transgenic hairy roots were used as a negative control. The differences of LR densities between 35S::GFP transgenic and untransformed roots were not significant (Table S1). After genotyping, a total of $4635 S:: G m I D L 2 a$ and $4935 S:: G m I D L 4 a$ transgenic hairy roots were obtained through Agrobacterium rhizogenes-mediated transformation, in which 34 35S::GmIDL2a and 31 35S::GmIDL4a hairy roots were, upon inspection, found to have aberrant phenotypes, respectively (Table S2, Figure 3A,B). The LRs (5-6 cm in length) were more luxuriant in 35S::GmIDL2a- and 35S::GmIDL4a-overexpressing roots than in the 35S::GFP control (Figure 3A). Compared with the 35S::GFP control, the LR densities of 35S::GmIDL2a and 35S::GmIDL4a hairy roots were increased significantly (Figure 3B), indicating the role of GmIDL2a and GmIDL4a in LR emergence.

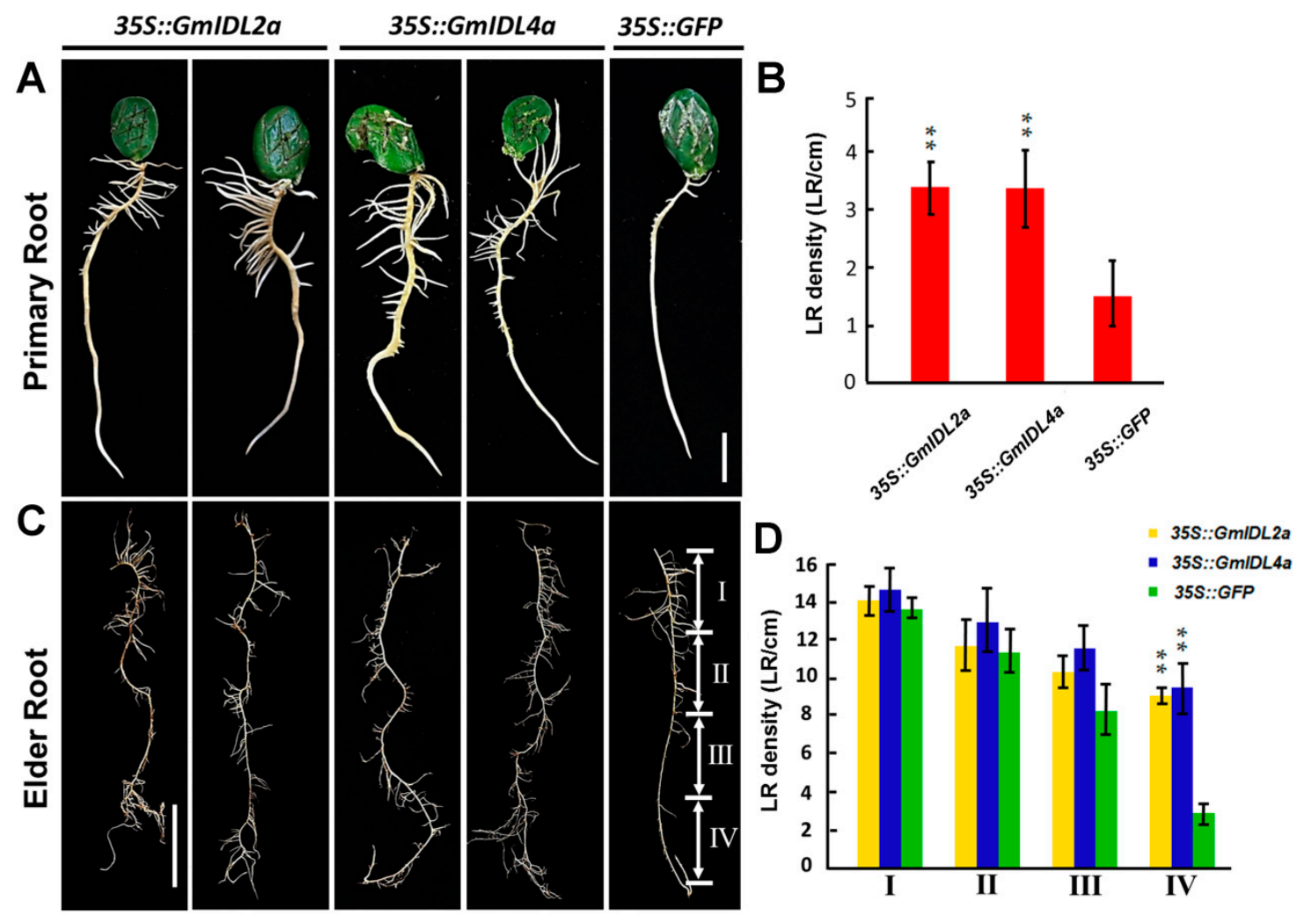

Figure 3. Morphological and statistical analysis of 35S::GmIDL2a, 35S::GmIDL4a, and 35S::GFP primary and elder roots. (A) Morphologies of 35S::GmIDL2a, 35S::GmIDL4a, and 35S::GFP primary hairy roots; bar = $1 \mathrm{~cm}$; (B) LR densities of 35S::GmIDL2a, 35S::GmIDL4a, and 35S::GFP primary hairy roots; (C) morphologies of 35S::GmIDL2a, 35S::GmIDL4a, and 35S::GFP elder roots (with length more than $20 \mathrm{~cm}$ ). I, II, III, and IV indicate four root segments; bar = $5 \mathrm{~cm}$; and (D) LR densities of 35S::GmIDL2a, 35S::GmIDL4a, and 35S::GFP in each segment of elder hairy roots. Student's $t$-test, ${ }^{* *} p<0.01$.

To elucidate whether the increased LR densities in 35S::GmIDL2a and 35S::GmIDL4a transformants reflected the differences in the rates of LRP initiation or emergence, elder hairy roots with a length of more than 20 centimeters were investigated. As shown in Figure 3C, from the top of the root (segment I) to the root tip (segment IV), the hairy roots were divided into four isometric segments. Morphological analysis revealed that there were no obvious differences in LR densities between the 35S::GmIDL2a, 35S::GmIDL4a, and 35S::GFP hairy roots from segment I to segment III (Figure 3C). By contrast, the LR densities in segment IV of the 35S::GmIDL2a and 35S::GmIDL4a hairy roots were 
significantly higher than those in the 35S::GFP control (Figure 3D), indicating an accelerated LR emergence in the 35S::GmIDL2a and 35S::GmIDL4a hairy roots. The statistical data also supported the morphologies. These results suggested that GmIDL2a and GmIDL4a facilitate LRP penetration through the overlaying cell layers during LR emergence.

\subsection{Overexpression of GmIDL2a and GmIDL4a Leads to Aberrant Cell Layer Separation}

As development continued, elder hairy roots with overexpression of GmIDL2a and GmIDL4a were also inspected for other changes in morphology. In 35S::GmIDL2a- and 35S::GmIDL4a- overexpressing roots, abnormal rifts which presumably experienced cell layer separation appeared in the elder roots. This phenotype suggested that the cell wall degradation appeared to have less control and restriction (Figure 4A-D). Infrequently, the overexpression of GmIDL2a and GmIDL4a even led to the fragmentation of the epidermis and an incompact type of the cortical cells (arrow in Figure 4C). We suspected that the adhesive force in these cell layers was insufficient to keep the epidermis adherence in place where cell wall degradation took place. As expected, these abnormal morphologies cannot be observed in the 35S::GFP control (Figure 4E). This phenomenon reflected the functions of GmIDL2 $a$ and GmIDL4a in intercellular adhesion and overlaying cell layer separation.

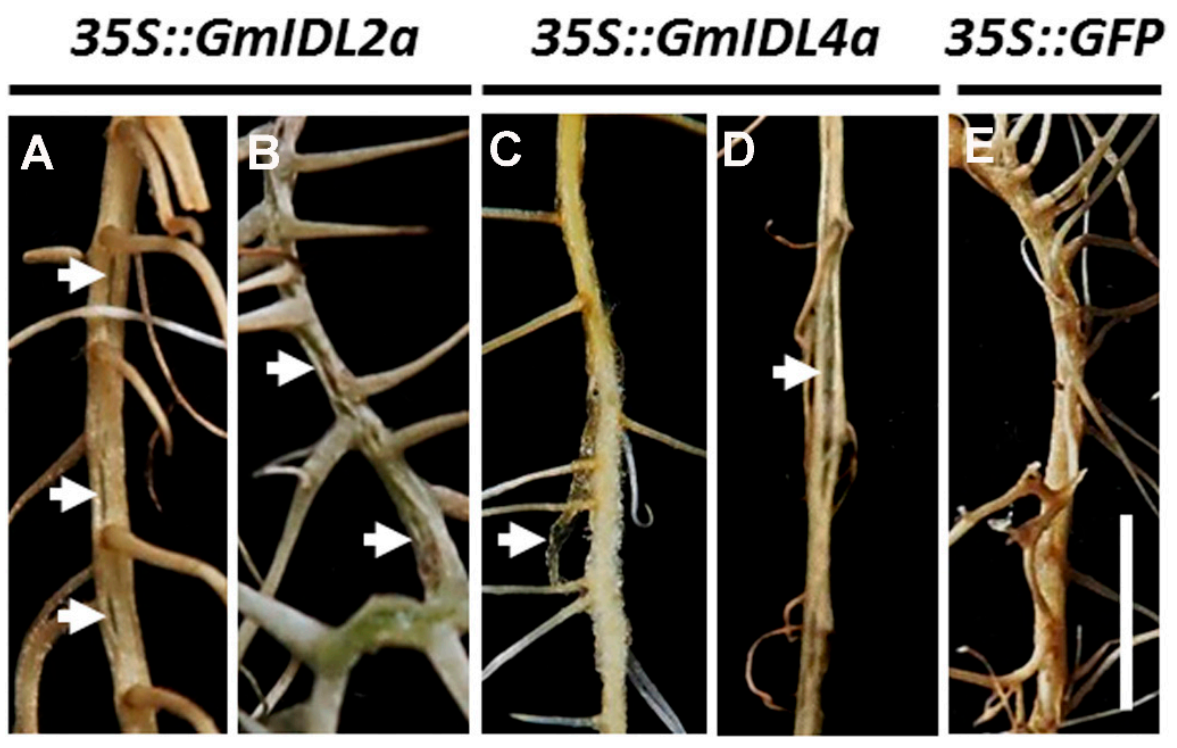

Figure 4. Aberrant morphologies of the 35S::GmIDL2a and 35S::GmIDL4a hairy roots. (A,B) Morphologies of 35S::GmIDL2a hairy roots; (C,D) morphologies of 35S::GmIDL4a hairy roots; and (E) normal root morphology of the 35S::GFP control. Arrows indicate aberrant rifts. Scale bar $=1 \mathrm{~cm}$.

Because LR morphogenesis was dependent on the overlaying tissues [15], we further observed the morphologies of the embedded LRPs and emerged LRs by cross section. The foreparts of the LRPs of 35S::GFP hairy roots were narrow and sharp (Figure 5A), while the 35S::GmIDL2a and 35S::GmIDL4a hairy roots were wider and more obtuse (Figure $5 B, C$ ). The overlaying tissues of the embedded LRPs were compact in the 35S::GFP control (Figure 5A). By contrast, the cell layers against the primordia were broken in the 35S::GmIDL2a and 35S::GmIDL4a hairy roots, suggesting an accelerated cell wall degradation (Figure 5B,C). The adhesion between the cell layers of the primary roots and emerged LRs were normal in the 35S::GFP hairy roots (Figure 5D). However, the cell layers of the primary roots were separated from the emerged LRs when GmIDL2a and GmIDL4a were overexpressed in hairy roots (Figure 5E,F). These results indicated that the overexpression of GmIDL2a and GmIDL4a led to abnormal cell layer disintegration, which appear as rifts in their morphology (Figure 4A,B,D). 


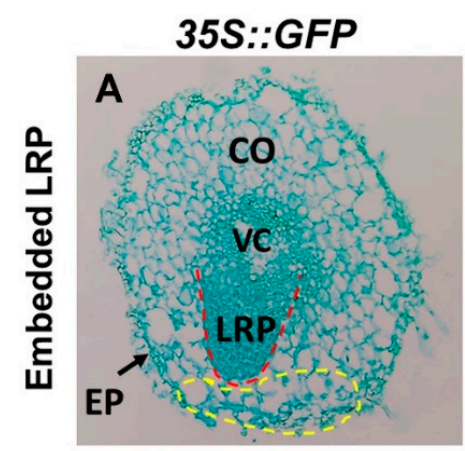

35S::GmIDL2a
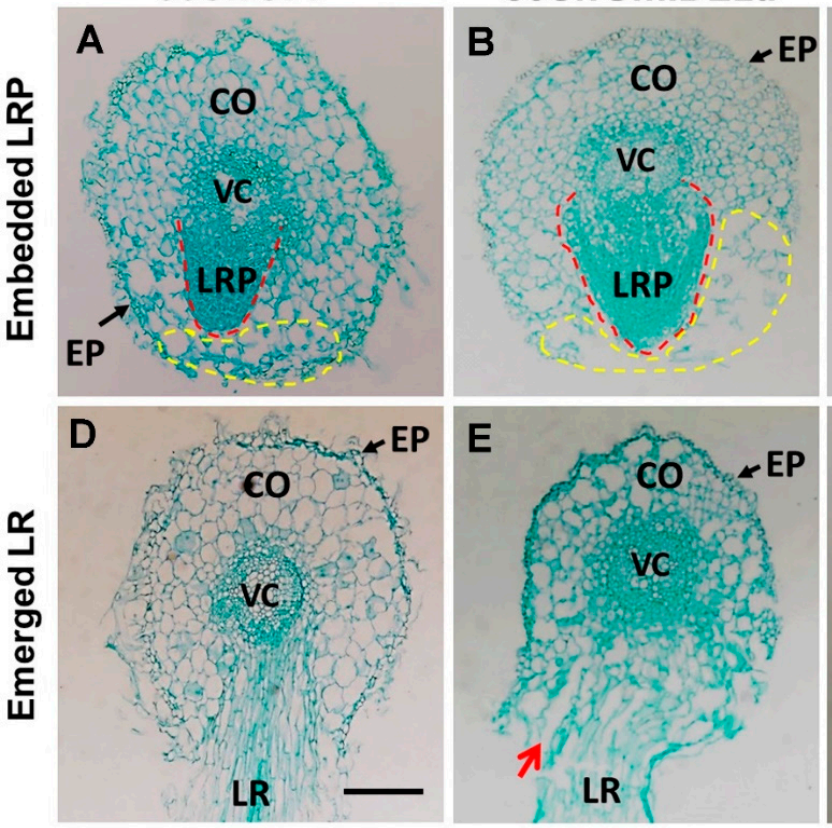

35S::GmIDL4a
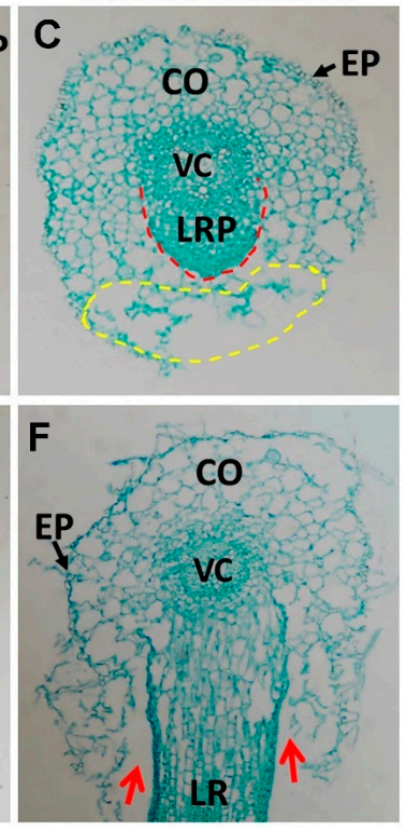

Figure 5. Histological sections of 35S::GFP, 35S::GmIDL2a, and 35S::GmIDL4a hairy roots. (A-C) Embedded LRPs of 35S::GFP (A), 35S::GmIDL2a (B), and 35S::GmIDL4a (C) hairy roots; (D-F) emerged LRs of 35S::GFP (D), 35S::GmIDL2a (E), and 35S::GmIDL4a (F) hairy roots. Yellow dashed lines indicate the overlaying tissues of embedded LRPs; Red dashed lines indicate the LRPs; Red arrows indicate the abnormal cell layer separation; EP, epidermal cells; CO, cortical cells; VC, vascular cylinder; LR, lateral root. Scale bar $=200 \mu \mathrm{m}$.

\subsection{GmIDL2a and GmIDL4a Regulate the Expression of CWR Genes during LR Emergency}

Plant cell wall degradation involves initial cell wall loosening through enzymes such as EXPs and XTHs [7,8]. The loosening of the cell wall facilitates the entrance of cell wall degradation enzymes, such as PGs, which hydrolyze pectins, the major component of plant cell walls. Because the aberrant phenotypes indicated ectopic cell wall degradation, we investigated the expression levels of representative members of the CWR genes. In plants, the CWR genes EXPs, XTHs, and PGs belong to multigene families [29-31]. In the present study, four EXPs, five XTHs, and four PGs with high expression levels in roots were selected for further analysis. Real-time qRT-PCR results showed that the expressions of the selected EXPs, XTHs, and PGs in the 35S::GmIDL2 $a$ and 35S::GmIDL4a hairy roots were higher than those in the 35S::GFP control (Figure 6A-C). These results suggested that GmIDL2a and GmIDL4a regulate the expression of CWR genes during LR emergence.

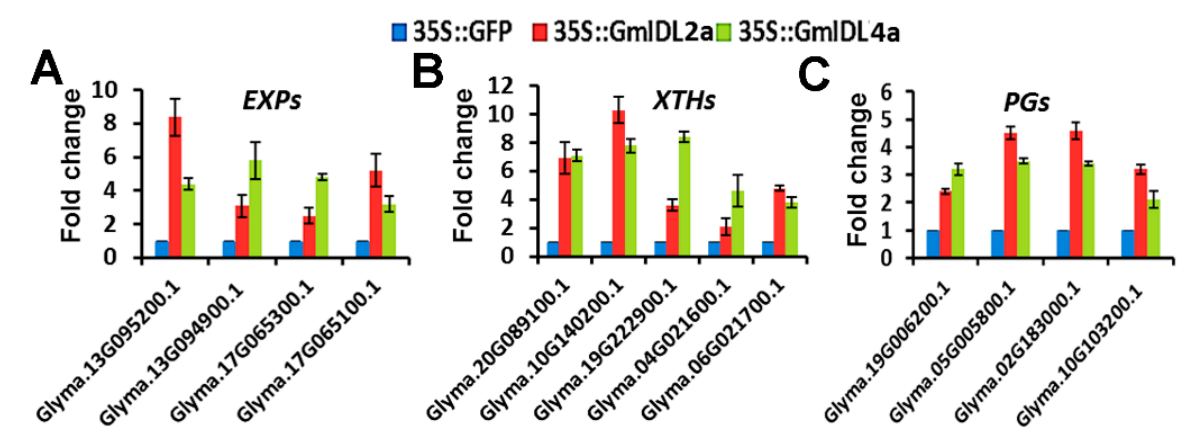

Figure 6. Expression of some members of EXPs, XTHs, and PGs genes in 35S::GmIDL2a, 35S::GmIDL4a, and 35S::GFP hairy roots. (A) Relative expressions of EXPs; (B) relative expressions of XTHs; and (C) relative expressions of $P G s$. 


\section{Discussion}

\subsection{GmIDL Genes Are Ubiquitous and Redundant in Multiple Plant Species}

It has been reported that IDA is not only associated with floral organ abscission, but also controls the separation step of the overlaying tissues during LR emergence, suggesting the vital role of the IDA ligand in the IDA-HAE/HSL2 signaling pathway [18,32]. Besides IDA in Arabidopsis, the other five IDL genes have been identified through a tBLASTn search using the conserved EPIP motif of the IDA protein, which is sufficient to induce abscission through its receptor HAE/HAL2 [18,28]. Similar to IDA, AtIDL1, AtIDL2, and AtIDL5 were also expressed in inflorescence; AtIDL1 and AtIDL4 were both expressed in roots [18]. In the present study, we performed a BLAST search in some representative monocotyledon and dicotyledon species. The results illustrated that IDL genes are ubiquitous in multiple plant species (Figure 1B). In soybean, there are a total of twelve GmIDL genes extant in the genome. The twelve GmIDL proteins can be divided into two groups. Each group contains two members which are similar in protein sequence (Figure 1A). One explanation for this phenomenon is that the diploid ancestor of soybean $(n=11)$ underwent aneuploid loss $(n=10)$ and subsequent polyploidization [33]. In the soybean genome, many genes existed as multigene families. Therefore, the functions of GmIDL proteins may be redundant in both floral organ abscission and LR emergence. Our data support this hypothesis. There are three GmIDL genes that were expressed in roots, of which GmIDL2 $a$ and GmIDL4a are highly expressed [Figure 2A]. Functional analysis results show that both GmIDL2 $a$ and GmIDL4a regulate the expression of CWR genes during LR emergence (Figures 4 and 6).

\subsection{IDA and IDL Act as Ligands and Are Expressed Specifically at the Site Where Cell Separation Occurs}

In Arabidopsis, more than 1000 genes encode putative small peptides [34], suggesting that small peptides may play important roles in plant growth and development. Some small peptides function as ligands and play a role with its receptor in cell-to-cell communication through ligand-receptor interaction [24]. However, few signaling models have been identified by genetic and/or biochemical strategies [35]. The IDA-HAE/HSL2 signaling pathway is well known for its function in controlling floral organ abscission and LR emergence [18,24]. Previous studies have demonstrated that the $I D A$ and IDL genes were expressed and restricted in both AZs and the cell layers overlaying new LRPs $[18,24]$. In normal conditions, plant cells attach to adjacent cells throughout their life cycle, but in some situations, it is crucial for plants to break this attachment, and thus cell separation occurs [36]. There are two consequences for cell separation. The beneficial result is the shedding of its needless organs such as flowers, fruits, or leaves; the adverse is the exposure of the separated cells to the outer environment, which puts plants at risk of pathogenic microorganism infection. Interestingly, plants have evolved some protection mechanisms, one of which is the expression pattern of the genes involved in abscission, such as IDA and IDL [18,24,25]. The expression of IDA and IDL is restricted in AZs where organ shedding occurs; consequently, this protects other tissues from pathogen infection. For LR emergence, IDA and IDL are specifically expressed in cell layers overlaying new LRPs ([18,24], Figure 2D,E). In soybean, consistent results were obtained by Tucker et al. [27]. As a result, cell wall breakdown only happens in the cells that face the LRPs. By contrast, the constitutive expression of GmIDL2a and GmIDL4a driven by CaMV $35 S$ promoters in hairy roots leads to the appearance of ectopic rifts, suggesting that excessive cell wall degradation occurred (Figure 4). In the soybean genome, there are two copies of each GmIDL gene (Figure 1). Although they are similar in amino acid sequence, the biological functions may be different. GmIDL2a/GmIDL4 and GmIDL4a/GmIDL8 are the two subfamilies of the GmIDL gene family; however, only GmIDL2a and GmIDL4a are highly expressed in roots (Figure $2 \mathrm{~A}, \mathrm{C}$ ). 


\subsection{GmIDL Signaling Pathways Are Involved in Cell Wall Degradation through Regulating CWR Gene Expressions}

In this work, we have demonstrated that LRs of the 35S::GmIDL2a and 35S::GmIDL4a transgenic hairy roots find it easier to penetrate from the primary roots (Figure 3A). LRs originate from pericycle cells $[37,38]$; therefore, the emergence of LRs out of the overlaying tissues is the result of cell division of the LRPs [15]. In addition, the emergence of LRs is dependent on the cell layers overlaying the LRPs, because the properties of the overlaying tissues disrupt LRP morphogenesis [15]. In the present study, we found that the foreparts of the 35S::GmIDL2a and 35S::GmIDL4a hairy roots were wider and more obtuse. The control LRPs were narrow and sharp. Conversely, due to the restriction of the compact cell layers, LRPs in 35S::GFP hairy roots were narrow and sharp (Figure 5A-C). For LR emergence, cell wall loosening and degradation are two critical steps [25]. In these processes, enzymes such as XTHs and EXPs are responsible for cell wall loosening, which facilitates the access of cell wall degradation enzymes such as PGs $[13,14]$. The PGs, important components in cell separation events, are the enzymes that hydrolyze pectins. Previous results suggest that the IDA-HAE/HAL2 signaling model is involved in the regulation of CWR genes [13]. Both ida mutants and hae mutants show deficiencies in cell wall degradation, because the expressions of CWR genes are more decreased than those of the wild type [24]. Similarly, GmIDL2a and GmIDL4a are also involved in the regulation of soybean CWR genes, which is the last step for cell wall degradation (Figure 6). However, the regulation mechanism of the IDA signaling model is not fully understood. Therefore, other factors related to signal transduction must exist in or out of this model. At present, it has been demonstrated that mitogen-activated protein kinase 4 (MKK4), MKK5, MAPK3, and MAPK6 act downstream of IDA/HAE through genetic studies and in vitro kinase assays [32]. The knotted-like homeobox genes brevipedicellus (BP)/knotted-like genes from Arabidopsis thaliana 1 (KNAT1) are proposed to act downstream in the IDA-HAE/HAL2 abscission pathway [39]. In conclusion, organ abscission and LR emergence are complicated physiological processes that are involved in programmed changes in cellular adhesion. More research, other than on IDA and HAE, should be performed to fully understand plant organ abscission.

\section{Materials and Methods}

\subsection{Bioinformatic Analysis}

IDL proteins were identified in the proteomes of seven species using the sequence of IDA (NP564941) and are available at the website (www.phytozome.net). Proteins which share high similarities with the EPIP motif and belong to small peptides were deemed as IDL proteins. Sequence alignment was performed using DNAMAN software. A cladogram was prepared using the software MEGA 5.0. The typical genes encoding XTH (AT4G25810), EXP (AT4G01630), and PG (AT5G14650 and AT2G41850) in soybean were identified using the Arabidopsis XTH (AT4G25810), EXP (AT4G01630), and PG (AT5G14650 and AT2G41850) protein sequences in the soybean genome, and then the expression data were illustrated in the BLAST result (https:/ / phytozome.jgi.doe.gov/pz/portal.html). Genes with high expression levels in roots were selected for CWR gene expression level detection.

\subsection{Soybean Root Treatment, PCR, RT-PCR, and Real-Time qRT-PCR}

Soybean seeds (XIAOLIDOU) were sterilized with 70\% ethanol for two minutes and then washed with sterile water five times. Furthermore, the seeds were sterilized with $0.1 \% \mathrm{HgCl}_{2}$ for $6 \mathrm{~min}$, then washed five times with sterile water for $2 \mathrm{~min}$. The sterile seeds were tiled on sterile filter papers to perform the water air-drying, then incubated on $1 / 2 \mathrm{MS}$ basal medium with the hilums upward at $28{ }^{\circ} \mathrm{C}$ under a light/dark period of $16 / 8 \mathrm{~h}$. The germinated seeds with a root length between three and four centimeters were used for phytohormone treatment or cut down with a scalpel for RNA extraction. 
For phytohormone treatment, soybean seeds were transferred to a MS medium supplemented with $1 \mu \mathrm{M}$ IAA and treated for $6,12,18$, and $24 \mathrm{~h}$, respectively. The treated roots were harvested for RNA extraction and qRT-PCR assay.

Genomic DNA was extracted from hairy roots as described previously [40]. Total RNA was extracted using the total RNA isolation kit (Promega, Madison, WI, USA) according to the manufacturer's recommendations. A total of $3 \mu \mathrm{g}$ total RNA was reverse transcripted into cDNA using the reverse transcription kit (Promega, Madison, WI, USA) following the manufacturer's instructions.

PCR and RT-PCR were performed with the gene-specific primers (Table S3) according to the amplification parameters: $95^{\circ} \mathrm{C}$ for $5 \mathrm{~min}, 94^{\circ} \mathrm{C}$ for $1 \mathrm{~min}, 56{ }^{\circ} \mathrm{C}$ for $1 \mathrm{~min}$, and $72{ }^{\circ} \mathrm{C}$ for $30 \mathrm{~s}$ repeated for 30 cycles, and a final extension for $10 \mathrm{~min}$ at $72{ }^{\circ} \mathrm{C}$. Reactions were carried out with Taq DNA polymerase (Takara, Dalian, China) in a Biometra Thermo cycler (TG-96; Biometra, Horsham, PA, USA), and the PCR products were analyzed on $0.8 \%$ agarose gels. Soybean actin gene (TC204137) was used as a reference gene.

Real-time qRT-PCR was performed in a $20 \mu \mathrm{L}$ reaction system containing SYBR GREEN reaction mixture (Takara, Dalian, China), $10 \mu \mathrm{M}$ of each primer, and $50 \mathrm{ng}$ cDNA. The sequences of the primers were listed in Table S2. Amplification was carried out on a Bio-Rad Real-Time System CFX96TM C1000 thermal cycler (Hercules, CA, USA) following the manufacturer's instructions.

\subsection{Plasmid Construction and Soybean Transformation}

The promoter fragments of GmIDLs, which included $2 \mathrm{~kb}$ upstream of the ATG of GmIDLs, were amplified using primer pairs $\mathrm{P}_{\text {IDL3 } 3} \mathrm{~F} / \mathrm{P}_{\text {IDL3 }} \mathrm{R}$ and $\mathrm{P}_{\text {IDL7 }} \mathrm{F} / \mathrm{P}_{\text {IDL7 }} \mathrm{R}$ with HindIII and BamHI restriction endonuclease sites at its $5^{\prime}$ and $3^{\prime}$ ends, respectively. The PCR products were cloned into the pMD19T vector (Takara, Japan) for sequencing. The correct clones were introduced into pBI121 to generate GmIDL::GUS constructs.

GmIDL encoding sequences were amplified using primer pairs GmIDL2aOEF/GmIDL2aOER and GmIDL4aOEF/GmIDL4aOER, respectively. The open reading frames of GmIDL2a and GmIDL4a were inserted into pBI121 under the control of CaMV $35 S$ promoters. The constructs were transferred into Agrobacterium rhizogenes strain R599 for soybean transformation.

Sterile soybean seeds were cultured on $1 / 2$ MS medium and grown at $28{ }^{\circ} \mathrm{C}$ under a light/dark period of $16 / 8 \mathrm{~h}$. The green cotyledons were used as explants for soybean hairy root transformation. The cotyledons were cut from soybean shoots and carved the adaxial surface to increase transformation efficiency. The Agrobacterium rhizogenes strain harboring binary vectors were cultured to $\mathrm{OD}_{600}=0.6-0.8$ for bacterium collection. The pellet was resuspended to $\mathrm{OD}_{600}=0.1$ with MS salts supplemented with $0.1 \mathrm{mg} / \mathrm{L}$ 1-naphthlcetic acid, $2 \mathrm{mg} / \mathrm{L}$ 6-benzylaminopurine, and $10 \mu \mathrm{M}$ acetosyringone ( $\mathrm{pH}$ 5.2). The explants were infected by Agrobacterium rhizogenes for ten minutes, then the excess bacterial solution was removed by sterile filter papers. The air-dried explants were cocultured on an infection medium (MS $+10 \mu \mathrm{M}$ acetosyringone, pH 5.2) at $25^{\circ} \mathrm{C}$ in dark conditions for three days. The cocultured explants were then transferred to sterile conical flask and flushed with sterile water several times until the water was clear and pellucid. Then, the explants were transferred to hairy root-inducing medium (MS $+250 \mathrm{mg} / \mathrm{L}$ cefertaxin, $\mathrm{pH}$ 5.8) for hairy root induction.

\subsection{GUS Histochemical Analysis}

Hairy roots were cut from cotyledon explants and used for GUS staining assay. GUS staining was performed using the chromogenic substrate 5-bromo-4-chloro-3-indolyl- $\beta$-D-glucuronic acid (X-gluc; Bio Vectra, Oxford, CT, USA). Hairy roots were incubated with GUS staining buffer ( $1 \mathrm{mM} \mathrm{X-gluc}$ solution in $0.5 \mathrm{M}$ EDTA, $5 \mathrm{mM} \mathrm{FeK}_{3}(\mathrm{CN})_{6}, 5 \mathrm{mM} \mathrm{K}_{4} \mathrm{Fe}(\mathrm{CN})_{6}$, and $0.1 \%$ Triton $\mathrm{X}-100$ in $100 \mathrm{mM}$ sodium phosphate buffer ( $\mathrm{pH} 7.0)$ ) at $37^{\circ} \mathrm{C}$ for $12 \mathrm{~h}$. Following alcohol decolorization, the samples were photographed under a microscope (Olympus SEX16, Tokyo, Japan). 


\subsection{Cross-Section and Microscopy}

Wild-type and transgenic hairy roots were fixed in F.A.A ( $5 \%$ acetic acid, $5 \%$ formaldehyde solution, and $63 \%$ ethanol) at $4{ }^{\circ} \mathrm{C}$ for $48 \mathrm{~h}$. After dehydration in a series of ethanol concentrations, the samples were vitrified in a series of ethanol dimethylbenzene concentrations and then embedded in paraffin. The embedded blocks were sectioned with a microtome (Leica, Solms, Germany) at a thickness of $10 \mu \mathrm{m}$. The sections were stained with $0.5 \%$ fast green and observed with a microscope (Olympus BX51, Tokyo, Japan).

\subsection{Statistical Analysis}

For LR density assay, a total of 30 transgenic hairy roots from each construct's transformants were collected for statistical analysis. The hairy root density was defined as the average LR number per centimeter of taproot. The significance of difference was tested by the Student's $t$-test $(* * p<0.01)$.

Supplementary Materials: Supplementary materials can be found at http:/ / www.mdpi.com/1422-0067/19/8/ 2262/s1.

Author Contributions: C.L. and F.L. conceived and designed the experiments; C.L. and W.M. performed the experiments; C.Z. and M.C. analyzed the data; F.C., K.L. and M.F. contributed reagents/materials/analysis tools; C.L. wrote the paper.

Acknowledgments: This work was supported by the Natural Science Foundation of China (Grant No. 31401462).

Conflicts of Interest: The authors declare no conflict of interest.

\section{Abbreviations}

$\begin{array}{ll}\text { LR } & \text { Lateral root } \\ \text { LRP } & \text { Lateral root primordium } \\ \text { XTHs } & \text { Xyloglucan endotransglucosylase Hydrolases } \\ \text { EXPs } & \text { Expansins } \\ \text { PGs } & \text { Polygalacturonases } \\ \text { AZ } & \text { Abscission zone } \\ \text { CWR genes } & \text { Cell wall remodeling genes }\end{array}$

\section{References}

1. Péret, B.; Rybel, B.D.; Casimiro, I.; Benková, E.; Swarup, R.; Laplaze, L.; Beeckman, T.; Bennett, M.J. Arabidopsis lateral root development: An emerging story. Trends Plant Sci. 2009, 14, 399-408. [CrossRef] [PubMed]

2. Lavenus, J.; Goh, T.; Roberts, I.; Guyomarc'h, S.; Lucas, M.; Dmet, I.D.; Fukaki, H.; Beeckman, T.; Bennett, M.; Laplaze, L. Lateral root development in Arabidipsis: Fifty shades of auxin. Trends Plant Sci. 2013, 18, 450-458. [CrossRef] [PubMed]

3. Dolan, L.; Janmaat, K.; Willemsen, V.; Linstead, P.; Poethig, S.; Roberts, K.; Scheres, B. Cellular organization of the Arabidopsis thaliana root. Development 1993, 119, 71-84. [PubMed]

4. Malamy, J.E.; Benfey, P.N. Organization and cell differentiation in lateral roots of Arabidopsis thaliana. Development 1997, 124, 33-44. [PubMed]

5. Casimiro, I.; Marchant, A.; Bhalerao, R.P.; Beeckman, T.; Dhooge, S.; Swarup, R.; Graham, N.; Inzé, D.; Sandberg, G.; Casero, P.J.; et al. Auxin transport promotes Arabidopsis lateral root initiation. Plant Cell 2001, 13, 843-852. [CrossRef] [PubMed]

6. Dubrovsky, J.G.; Rost, T.L.; Colón-Carmona, A.; Doerner, P. Early primordium morphogenesis during lateral root initiation in Arabidopsis thaliana. Planta 2001, 214, 30-36. [CrossRef] [PubMed]

7. Geldner, N.; Friml, J.; Stierhof, Y.D.; Jürgens, G.; Palme, K. Auxin transport inhibitors block PIN1 cycling and vesicle trafficking. Nature 2001, 413, 425-428. [CrossRef] [PubMed]

8. Friml, J.; Benková, E.; Blilou, I.; Wisniewska, J.; Hamann, T.; Ljung, K.; Woody, S.; Sandberg, G.; Scheres, B.; Jürgens, G.; et al. AtPIN4 mediates Sink-Driven auxin gradients and root patterning in Arabidopsis. Cell 2002, 108, 661-673. [CrossRef] 
9. De Smet, I.; Tetsumura, T.; De Rybel, B.; Frei dit Frey, N.; Laplaze, L.; Casimiro, I.; Swarup, R.; Naudts, M.; Vanneste, S.; Audenaert, D.; et al. Auxin-dependent regulation of lateral root positioning in the basal meristem of Arabidopsis. Development 2007, 134, 681-690. [CrossRef] [PubMed]

10. Parizot, B.; Roberts, I.; Raes, J.; Beeckman, T.; De Smet, I. In silico analyses of pericycle cell populations reinforce their relation with associated vasculature in Arabidopsis. Philos. Trans. R. Soc. B 2012, 367, 1479-1488. [CrossRef] [PubMed]

11. Rogg, L.E.; Lasswell, J.; Bartel, B. A gain-of-function mutation in IAA28 suppresses lateral root development. Plant Cell 2001, 13, 465-480. [CrossRef] [PubMed]

12. De Rybel, B.; Vassileva, V.; Parizot, B.; Demeulenaere, M.; Grunewald, W.; Audenaert, D.; Van Campenhout, J.; Overvoorde, P.; Jansen, L.; Vanneste, S.; et al. A novel aux/IAA28 signaling cascade activates GATA23dependent specification of lateral root founder cell identity. Curr. Biol. 2010, 20, 1697-1706. [CrossRef] [PubMed]

13. Cai, S.; Lashbrook, C.C. Stamen abscission zone transcriptome profiling reveals new candidates for abscission control: Enhanced retention of floral organs in transgenic plants overexpressing Arabidopsis ZINC FINGER PROTEIN2. Plant Physiol. 2008, 146, 1305-1321. [CrossRef] [PubMed]

14. Ogawa, M.; Kay, P.; Wilson, S.; Swain, S.M. ARABIDOPSIS DEHISCENCE ZONE POLYGALACTURONASE1 (ADPG1), ADPG2, and QUARTET2 are polygalacturonases required for cell separation during reproductive development in Arabidopsis. Plant Cell 2009, 21, 216-233. [CrossRef] [PubMed]

15. Lucas, M.; Kenobia, K.; Wangenheim, D.V.; Voß, U.; Swarupa, K.; De Smet, I.; Van Damme, D.; Lawrence, T.; Péret, B.; Moscardih, E.; et al. Lateral root morphogenesis is dependent on the mechanical properties of the overlaying tissues. Proc. Natl. Acad. Sci. USA 2013, 110, 5229-5234. [CrossRef] [PubMed]

16. Jinn, T.L.; Stone, J.M.; Walker, J.C. HAESA, an Arabidopsis leucine-rich repeat receptor kinase, controls floral organ abscission. Gene. Dev. 2000, 14, 108-117. [PubMed]

17. Liu, B.; Butenko, M.A.; Shi, C.L.; Bolivar, J.L.; Winge, P.; Stenvik, G.E.; Vie, A.V.; Leslie, M.E.; Tore Brembu, T.; Kristiansen, W.; et al. NEVERSHED and INFLORESCENCE DEFICIENT IN ABSCISSION are differentially required for cell expansion and cell separation during floral organ abscission in Arabidopsis thaliana. J. Exp. Bot. 2013, 64, 5345-5357. [CrossRef] [PubMed]

18. Butenko, M.A.; Patterson, S.E.; Grini, P.E.; Stenvik, G.E.; Amundsen, S.S.; Mandal, A.; Aalen, R.B. INFLORESCENCE DEFICIENT IN ABSCISSION Controls Floral Organ Abscission in Arabidopsis and Identifies a Novel Family of Putative Ligands in Plants. Plant Cell 2003, 15, 2296-2307. [CrossRef] [PubMed]

19. Wilmowicz, E.; Kućko, A.; Ostrowski, M.; Panek, K. INFLORESCENCE DEFICIENT IN ABSCISSION-like is an abscission associated and phytohormone-regulated gene in flower separation of Lupinus luteus. Plant Growth Regul. 2018, 85, 91-100. [CrossRef]

20. Stø, I.M.; Orr, R.S.S.; Fooyontphanich, K.; Jin, X.; Knutsen, J.M.B.; Fischer, U.; Tranbarger, T.J.; Nordal, I.; Aalen, R.B. Conservation of the abscission signaling peptide IDA during Angiosperm evolution: Withstanding genome duplications and gain and loss of the receptors HAE/HSL2. Front. Plant Sci. 2015, 6, 931. [CrossRef] [PubMed]

21. Estornell, L.H.; Wildhagen, M.; Pérez-Amador, M.A.; Talón, M.; Tadeo, F.R.; Butenko, M.A. The IDA peptide controls abscission in Arabidopsis and Citrus. Front. Plant Sci. 2015, 6, 1003. [CrossRef] [PubMed]

22. Ying, P.; Li, C.; Liu, X.; Xia, H.; Zhao, M.; Li, J. Identification and molecular characterization of an IDA-like gene from litchi, LcIDL1, whose ectopic expression promotes floral organ abscission in Arabidopsis. Sci. Rep. 2016, 6, 37135. [CrossRef] [PubMed]

23. Stenvik, G.E.; Butenko, M.A.; Urbanowicz, B.R.; Rose, J.K.C.; Aalen, R.B. Overexpression of INFLORESCENCE DEFICIENT IN ABSCISSION activates cell separation in vestigial abscission zones in Arabidopsis. Plant Cell 2006, 18, 1467-1476. [CrossRef] [PubMed]

24. Kumpf, R.P.; Shi, C.L.; Antoine Larrieu, A.; Stø, I.M.; Butenko, M.A.; Péret, B.; Riiser, E.S.; Bennett, M.J.; Aalen, R.B. Floral organ abscission peptide IDA and its HAE/HSL2 receptors control cell separation during lateral root emergence. Proc. Natl. Acad. Sci. USA 2013, 110, 5235-5240. [CrossRef] [PubMed]

25. Wang, X.; Hou, S.; Wu, Q.; Lin, M.; Acharya, B.R.; Wu, D.; Zhang, W. IDL6-HAE/HSL2 impacts pectin degradation and resistance to Pseudomonas syringae pv tomato DC3000 in Arabidopsis leaves. Plant J. 2017, 89, 250-263. [CrossRef] [PubMed]

26. Kim, J.; Yang, R.; Chang, C.; Park, R.; Tucker, M.L. The root-knot nematode Meloidogyne incognita produces a functional mimic of the Arabidopsis INFLORESCENCE DEFICIENT IN ABSCISSION signaling peptide. J. Exp. Bot. 2018, 69, 3009-3021. [CrossRef] [PubMed] 
27. Tucker, M.L.; Yang, R. IDA-like gene expression in soybean and tomato leaf abscission and requirement for a diffusible stellar abscission signal. AoB Plants 2012, 2012, pls035. [CrossRef] [PubMed]

28. Stenvik, G.E.; Tandstad, N.M.; Guo, Y.; Shi, C.L.; Kristiansen, W.; Holmgren, A.; Clark, S.E.; Aalen, R.B.; Butenkoa, M.A. The EPIP peptide of INFLORESCENCE DEFICIENT IN ABSCISSION is sufficient to induce abscission in Arabidopsis through the receptor-like kinases HAESA and HAESA-LIKE2. Plant Cell 2008, 20, 1805-1817. [CrossRef] [PubMed]

29. Li, Y.; Darley, C.P.; Ongaro, V.; Fleming, A.; Schipper, O.; Baldauf, S.L.; McQueen-Mason, S.J. Plant expansins are a complex multigene family with an ancient evolutionary origin. Plant Physiol. 2002, 128, 854-864. [CrossRef] [PubMed]

30. Liu, Y.; Liu, D.; Zhang, H.; Gao, H.; Guo, X.; Wang, D.; Zhang, X.; Zhang, A. The $\alpha$ - and $\beta$-expansin and xyloglucan endotransglucosylase/hydrolase gene families of wheat: Molecular cloning, gene expression, and EST data mining. Genomics 2007, 90, 516-529. [CrossRef] [PubMed]

31. Hadfield, K.A.; Bennett, A.B. Polygalacturonases: Many genes in search of a function. Plant Physiol. 1998, 117, 337-343. [CrossRef] [PubMed]

32. Cho, S.K.; Larue, C.T.; Chevalier, D.; Wang, H.; Jinn, T.L.; Zhang, S.; Walker, J.C. Regulation of floral organ abscission in Arabidopsis thaliana. Proc. Natl. Acad. Sci. USA 2008, 105, 15629-15634. [CrossRef] [PubMed]

33. Lackey, J.A. Chromosome numbers in the Phaseoleae (Fabaceae: Faboideae) and their relation to taxonomy. Am. J. Bot. 1980, 67, 595-602. [CrossRef]

34. Lease, K.A.; Walker, J.C. The Arabidopsis unannotated secreted peptide database, a resource for plant peptidomics. Plant Physiol. 2006, 142, 831-838. [CrossRef] [PubMed]

35. Butenko, M.A.; Vie, A.K.; Brembu, T.; Aalen, R.B.; Bones, A.M. Plant peptides in signalling: Looking for new partners. Trends Plant Sci. 2009, 14, 255-263. [CrossRef] [PubMed]

36. Roberts, J.A.; Elliot, K.A.; Gonzalez-Carranza, Z.H. Abscission, dehiscence and other cell separation processes. Annu. Rev. Plant Biol. 2002, 53, 131-158. [CrossRef] [PubMed]

37. De Smet, I.; Vanneste, S.; Inzé, D.; Beeckman, T. Lateral root initiation or the birth of a new meristem. Plant Mol. Biol. 2006, 60, 871-887. [CrossRef] [PubMed]

38. De Smet, I. Lateral root initiation: One step at a time. New Phytol. 2012, 193, 867-873. [CrossRef] [PubMed]

39. Shi, C.L.; Stenvik, G.E.; Vie, A.K.; Bones, A.M.; Pautot, V.; Proveniers, M.; Aalen, R.B.; Butenko, M.A. Arabidopsis Class I KNOTTED-Like homeobox proteins act downstream in the IDA-HAE/HSL2 floral abscission signaling pathway. Plant Cell 2011, 23, 2553-2567. [CrossRef] [PubMed]

40. Doyle, J.J.; Doyle, J.L. A rapid total DNA preparation procedure for fresh plant tissue. Focus 1990, 12, $13-15$. 